\title{
Enthesitis of the hands in psoriatic arthritis: an ultrasonographic perspective
}

\author{
Alen Zabotti ${ }^{1}$, Luca Idolazzi ${ }^{2}$, Alberto Batticciotto ${ }^{3}$, Orazio De Lucia ${ }^{4}$, Carlo Alberto Scirè ${ }^{5}$, \\ Ilaria Tinazzi ${ }^{6}$, Annamaria Iagnocco ${ }^{7}$
}

${ }^{1}$ Rheumatology Clinic, Department of Medical and Biological Sciences, University Hospital Santa Maria della Misericordia, Udine, ${ }^{2}$ Rheumatology Unit, University of Verona, Ospedale Civile Maggiore, Verona, ${ }^{3}$ Rheumatology Unit, L. Sacco University Hospital, Milan, ${ }^{4}$ Department of Rheumatology, ASST Centro traumatologico ortopedico G. Pini - CTO, Milan, ${ }^{5}$ Department of Medical Sciences, Section of Rheumatology, University of Ferrara, Ferrara, ${ }^{6}$ Unit of Rheumatology, Ospedale Sacro Cuore, Negrar, Verona, ${ }^{7}$ Dipartimento di Scienze Cliniche e Biologiche, Università degli Studi di Torino, Turin, Italy

\begin{abstract}
Psoriatic arthritis is a systemic inflammatory disease in which enthesitis and dactylitis are two of the main hallmarks of the disease. In the last years, ultrasonography is increasingly playing a key role in the diagnosis of psoriatic arthritis and ultrasonography of the entheses, particularly of the lower limbs, is commonly used to assess patients with that disease. New advancements in ultrasound equipment using high frequencies probes allowed us also to identify and characterize the involvement of the entheses of the hand in psoriatic arthritis, confirming the results of the experimental models of the disease and the theory of the sinovial-entheseal complex, even in small joints.
\end{abstract}

Keywords: ultrasonography; psoriatic arthritis; enthesitis; seronegative arthritis; synovio-entheseal complex

\section{Introduction}

Psoriatic Arthritis (PsA), usually included in the Spondyloarthritis (SpA) group, can affect different articular structures, from bone to soft tissues (e.g. tendons, entheses, and bursae) and enthesitis seems to be the earliest lesion in animal models of SpA [1,2]. From a clinical point of view, in 1971 Ball et al firstly described enthesitis in SpA [3]. Two decades later McGonagle et al demonstrated the concept of the involvement of the synovio-entheseal complex (SEC) and enthesis-related inflammation as an early event in PsA and SpA and its diagnostic use-

Received 11.07.2017 Accepted 22.08.2017

Med Ultrason

2017, Vol. 19, No 4, 438-443

Corresponding author: Prof. Annamaria Iagnocco

Dipartimento di Scienze Cliniche e Biologiche, Università degli Studi di Torino

Azienda Ospedaliero-Universitaria San Luigi

Gonzaga

Regione Gonzole 10,

10043 Orbassano Torino, Italy

E-mail: annamaria.iagnocco1@gmail.com fulness to differentiate PsA from Rheumatoid Arthritis (RA) $[4,5]$. The European League Against Rheumatism (EULAR) recommends the use of imaging in diagnosis and management of SpA and, in the last years, ultrasound (US) of the entheses, particularly of the lower limbs, has been increasingly applied in diagnostic studies on PsA [6,7]. Differential diagnosis in seronegative polyarthritis of the small joints is a challenge for clinicians and with potential diagnostic errors often occur. Recently, several studies have highlighted the role of hands US to assist the differential diagnosis between PsA and RA, demonstrating more severe extra-synovial involvement which includes the SEC in the former group [8-10]. In 2001, Benjamin et al proposed to distinguish entheses in classical and functional: in the latter fibrocartilage are in contact with bone but are not attached [11]. Functional entheses plays a key role in resisting shear and compressive mechanical stress of the entheseal organ $[4,12]$.

The aim of this pictorial essay is to describe and show the most common US findings of functional and classical enthesitis of the hands in patients with PsA. 


\section{Functional entheses}

The digits have several functional entheses associated with the presence of fibrocartilage to reduce mechanical stress. Here we report examples of hands functional enthesitis during PsA and the description of their US elementary lesions.

\section{Extensor tendon on metacarpophalangeal joint}

Despite the crucial role of tendon involvement in chronic inflammatory arthritis, most of US research has been focused on synovitis, some on tenosynovitis, and very few on the peritenonitis (PTI) [13]. Gutierrez et al described the presence of US detectable tendon thickening, echostructural hypoechogenicity, and hypoechoic swelling of the tissue surrounding the tendon, with or without peritendineal power Doppler (PD) signal at the level of tendons without synovial sheath [14]. One of the images they presented, showed the peritendinitis of the extensor digitorium tendons at the level of the metacarpophalangeal (MCP) joints, defined as hypoechogenicity at the level of peritenon with associated oedema of the surrounding soft tissues and PD signal [14]. The same group published in 2011 preliminary data on high frequency US of peritenon extensor tendon inflammation in PsA and RA patients. PTI, defined as a hypoechoic swelling of the soft tissue surrounding the extensor digitorum tendon (with or without peri-tendinous PD signal), was found in $65.8 \%$ of the clinically involved MCP joints of the PsA group and in none of the RA patients examined [9] (fig 1). At the level of the MCP joints the PTI pattern could be intended as a part of an inflammatory involvement of the SEC, consisting of a functional enthesis formed by extensor digitorum tendon, sesamoid fibrocartilage in the tendon as it crosses the MCP joints, superficial and deep peritendinous tissues, and joint synovial capsule [11] (fig 2). These data suggested the relevant potential role of US in the differential diagnosis between RA and PsA at MCP joints level [9], expecially in early disease [8]. Recent preliminary works underline that PTI is as frequent as intra-articular synovitis, that it can be a cause of MCPs swelling and that PD positivity at PTI is able to differentiate PsA development in early arthritis settings $[15,16]$.

\section{Digital pulleys}

The pulley system is essential for the accurate tracking of the flexor tendon and for maintaining the correct position of the tendon and bone across the joint and it provides a fulcrum to elicit flexion and extension. Considering the presence of fibrocartilage and its mechanical role, the pulley system is a typical example of functional enthesis. Currently, the most frequent US evaluation of the pulley system described in the literature is focused at

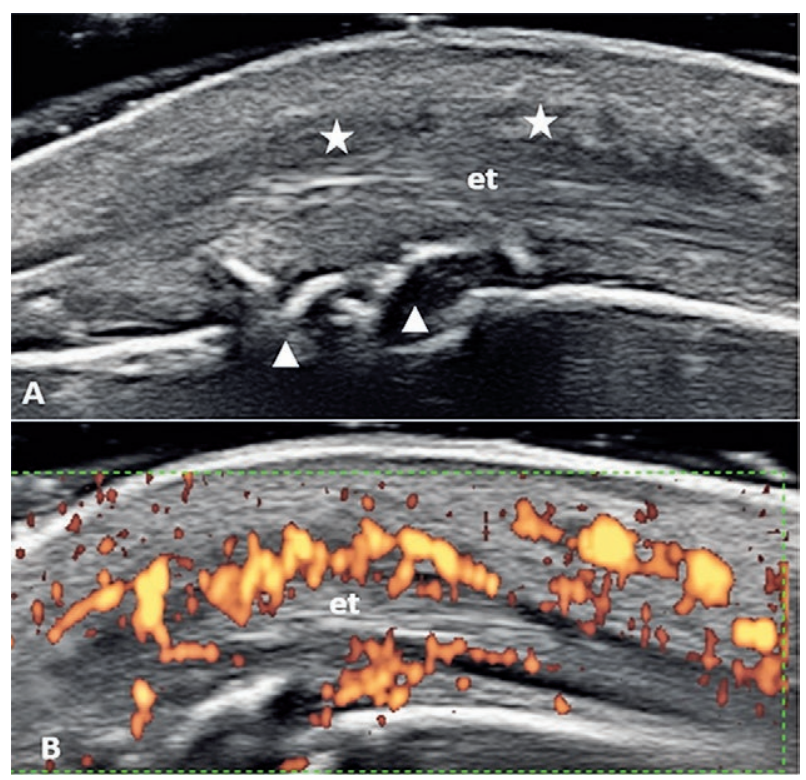

Fig 1. Dorsal longitudinal scan on MCP joint. A. Peritendon inflammation (white star) of the extensor digitorum tendon (et) with diffuse PD signal (B) and articular synovitis with osteoproliferation (white arrowheads).

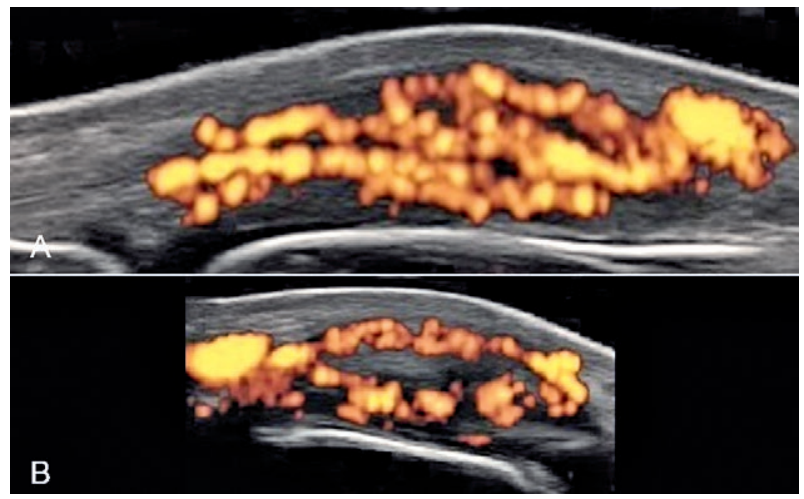

Fig 2. Dorsal scan on MCP joint (long - A and short axis - B). Peritendon inflammation of the extensor digitorum tendon with diffuse PD signal and active articular synovitis with extended PD signal.

the A1 pulley, located on the palmar aspect of the hand, at the level of the MCP joint [17]. For the thickness of that pulley, a cut-off value of $0.62 \mathrm{~mm}$ was proposed to discriminate between healthy and diseased structures [18]. Moreover, a positive correlation was found between US and intra-operative measurements of pulley thickness thus confirming the validity of US in the assessment of those structures [18]. In addition, non specific studies focused on the attachment of the pulley on the volar ridges of the proximal and middle phalanx. In inflammatory arthritis, pulleys can be affected during tenosynovitis as their loosening can be seen as secondary to the inflammatory process and this event could be a marker for dis- 


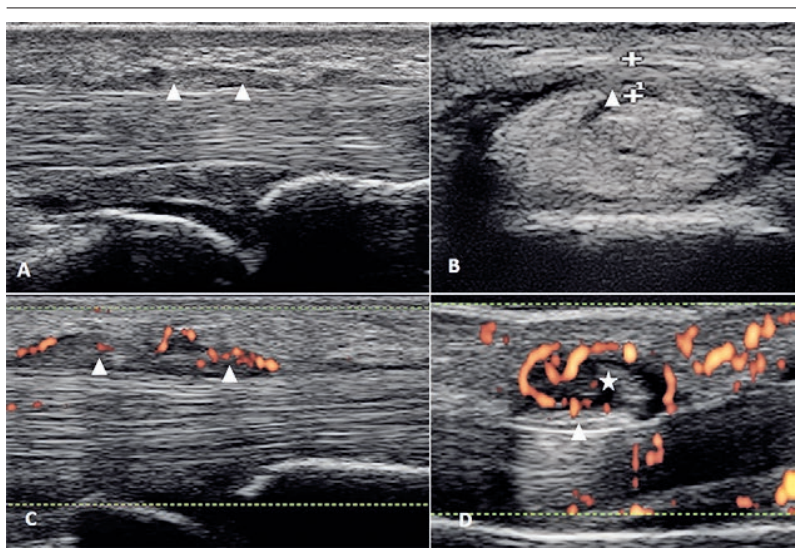

Fig 3. Volar scan on flexor tendon. Thickening of A1 pulley in long (A) and short axis (B) (white arrowheads). C and D are pulley images of a dactylitis. C Long axis of A1 pulley thickened and hypoechoic with PD signal (white arrowheads). D Long axis of A2 pulley (white arrowhead) with soft tissue oedema and PD signal around pulley (white star).

ease severity, affecting the function and other outcomes of the patients. Thus this feature could potentially be a marker of disease severity, affecting the hand function. Recently, Tan et al demonstrated by magnetic resonance imaging (MRI) inflammatory changes at digital pulleys and tendons in dactylitis in PsA patients, suggesting a form of functional enthesitis that could be related to the dactylitis process [19]. Moreover, Tinazzi et al showed pulley thickening in subjects affected by PsA compared with RA and healthy controls [20]. As depicted in figure 3 A1, A2 and A4 pulleys in PsA are easily detectable and measurable in longitudinal and transverse scan, but additional studies on early PsA patients are needed to ascertain whether these pulley changes may trigger PsA tenosynovitis or whether they represent a reactive process.

\section{Fibrous skeleton}

Dactylitis (also named sausage digit) is the clinical hallmark of PsA and it is an important predictor of structural damage [21]. In imaging studies (US and MRI), soft tissue oedema and flexor tenosynovitis are the two most important elementary lesions described in psoriatic dactylitis $[10,19,22]$. Overall, oedema of the soft tissues can be interpreted as an inflammation of the "fibrous skeleton" of the digit, which is made up of ligaments, fibrous capsular bands, palmar fasciae, and fibrous sheaths that attach to the bone or dermis [10]. However, this theory linking soft tissue oedema and functional enthesitis of the fibrous skeleton needs to be confirmed by pathology. Indeed, to date only a single case report focused on the histologic features of dactylitis: enthesitis and inflammation of tenosynovium were found to be prominent in that case and this inflammation reflected both a chronic $\mathrm{T}$ cell-rich inflammatory infiltrate and a stromal reaction

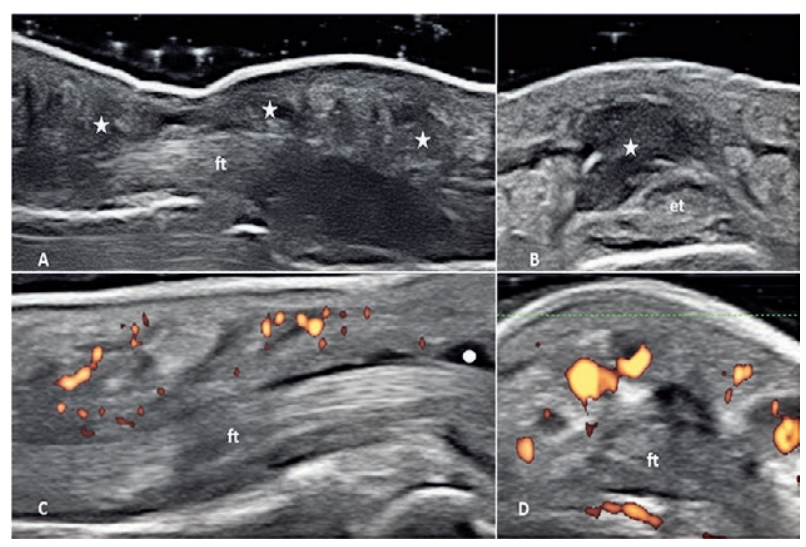

Fig 4. A Volar scan of the digit. Pseudotenosynovitis, diffuse soft tissue oedema (white star) without tenosynovitis of the flexor tendon (ft). B Dorsal scan on the MCP joint. Soft tissue oedema above extensor tendon (et). C and D Soft tissue oedema with intense PD signal around essudative flexor tenosynovitis (white point) in long axis (C) and short axis (D)

[23]. In US studies, soft tissue oedema of the digit was usually defined as a thickening of the soft tissue around the flexor tendon with an intense PD signal $[8,10,24]$ and it was also called by some authors as 'pseudotenosynovitis' [24,25] (fig 4). Such soft tissues thickening was also detected in fingers without clinical dactylitis, suggesting that this lesion could have a central role in the genesis of the psoriatic sausage digit. Moreover, Zabotti et al recently demonstrated that US detection of soft tissue oedema around the flexor tendon was highly specific for early PsA, if compared to early RA [8].

\section{Classical entheses}

The sites where tendons, ligaments and joint capsules attach to bone (i.e. classical enthesis) are the typical sites of inflammation in PsA. Currently, most of the US studies focused on the assessment of classical entheses at the level of the lower limbs in PsA for diagnosis and prognosis; however, other areas can be examined for this purpose, particularly the hand. An analysis of hands entesitis by US is reported as follows.

\section{Insertion of extensor tendon on distal interphalangeal joint and nail area}

Nail disease is often present in PsA patients and can be an early predictor of PsA [26,27]. There is a well known link between extensor tendon enthesopathy, distal interphalangeal (DIP) joint involvement, and nail pathology. As Tan et al elegantly showed by histology, the supporting fascia of the nail root is a continuation of the enthesis of the extensor tendon [28]. This could explain why PsA is associated with a local inflammation that involves both nail root and adjacent bone $[28,29]$. The US and clinical 
study of Aydin et al confirms the importance of extensor tendon distal enthesitis in the pathogenesis of nail disease with or without clinical arthritis [29], as previously suggested also by Ash et al [26]. Several studies showed that US is very useful to study enthesitis at the level of the distal attachment of the extensor tendon, as well as DIP joint synovitis and nail involvement $[28,29]$. The extensor tendon distal enthesis involvement can be seen by US as an abnormal hypoechoic tendon with loss of normal fibrillar architexture and/or thickened tendon at its distal phalanx attachment which are seen in two perpendicular planes and that may exhibit PD signals near to the bony cortex and/or bony changes, including enthesophytes, erosion or irregularities [30] (fig 5). The latest Outcome Measures in Rheumatology (OMERACT) definitions indicate for those abnormalities a location $\leq 2 \mathrm{~mm}$ from the bony cortex as a landmark of enthesitis in SpA [30]. However, they do not specify to which entheses this is applicable and if this can be considered as a characteristic abnormality also of small structures such as the distal enthesis of the hand extensor tendons. Furthermore, it is crucial also to distinguish findings related to inflammation from those due to structural damage as well as to use US in the differential diagnosis between a DIP involvement due to osteoarthritis or PsA. In this context, a clear and solid evidence from US studies is needed. In terms of nail imaging, US is able to show abnormalities occurring at the level of different components of the nail unit [14]. The possiblity to image by US the pathological changes of the nail, such as the loss of the normal trilaminar structure of the plate and the enhanced flow by PD $[14,31,32]$, in such sites could be a matter of speculation regarding the physiopathology of psoriatic disease, according with the nail/enthesis hypothesis [33] (fig 6). Another crucial aspect to consider in this context is the significance of possible nail pathology detected by US in patients without clinical involvement [34]. Finally, new advances in US equipment using high frequencies probes

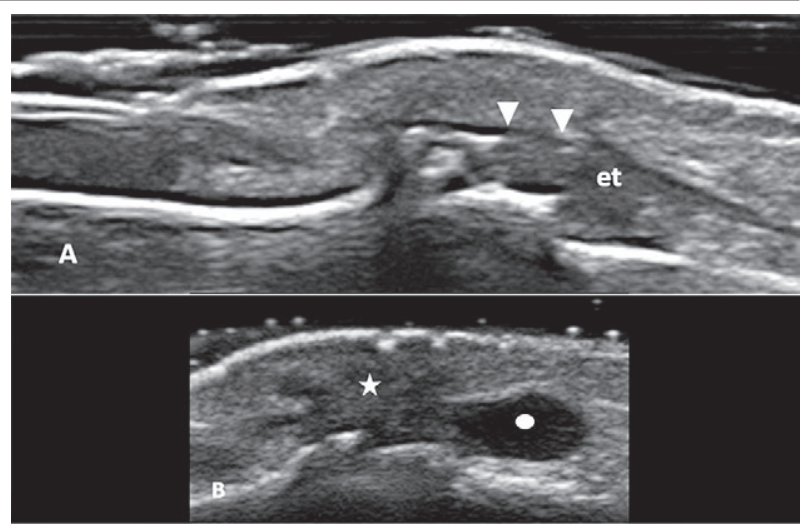

Fig 5. Longitudinal scan on the DIP joint. A. Thickening of the extensor tendon (et) with hypoechogenicity of tendon and enthesis (white arrowheads) and insertional entesophyte. B Soft tissue oedema (white star) and synovial effusion (white point) of the DIP joint.

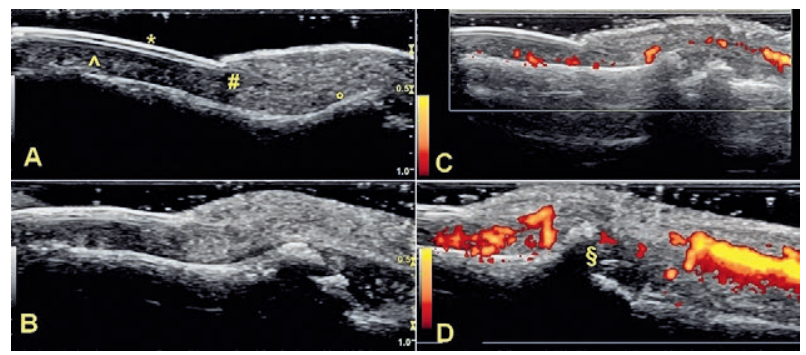

Fig 6. A Longitudinal scan of the nail: normal aspect. The nail plate $(*)$ is as a flawless trilaminar structure with sharp borders. Under the nail plate the nail bed $\left({ }^{\wedge}\right)$ appears as a ipo-isoechoic area under the nail plate and ending in the germinative matrix (\#). The insertion and enthesis of the extensor tendon is next to the germinal matrix $\left({ }^{\circ}\right)$. A picture of a psoriatic nail is in $\mathrm{B}$. The nail plate is altered, with loss of the trilaminar structure and thickening of the plate. The nail bed is thickened, with loss of homogeneity. In C the PD signal is depicted. Small vessels appear with a weak signal characterizing all the bed and some spots are also evident along the venule just next to the tendon. In $\mathrm{D}$ the signal is clearly increased and a single spot is also evident in the proximity of the fibrocartilage of the enthesis of the extensor tendon $(\S)$

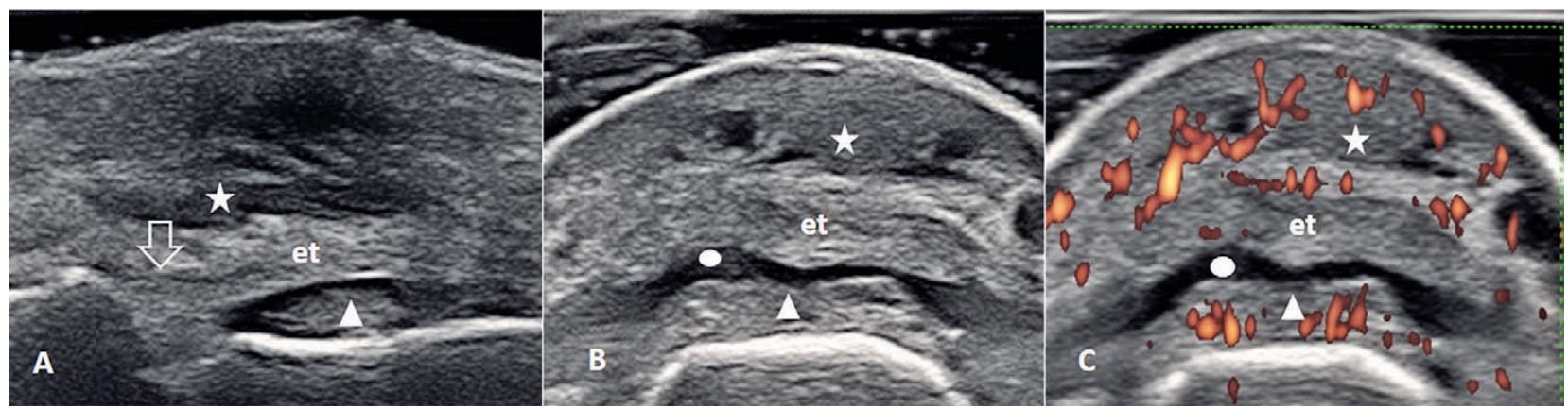

Fig 7. Dorsal scan on the PIP joint in long axis (A) and short axis (B) in GS and PD (C). Enthesitis of central slip of the extensor tendon (et) (arrow) with soft tissue oedema around the extensor tendon (white star) and articular synovitis (white arrowhead) and a small effusion (white point). 
(e.g. $\geq 22 \mathrm{MHz}$ ) might allow a better explanation of the link between the nail unit and the DIP joint, possibly revealing more specific alterations for psoriatic disease.

\section{Insertion of central slip of extensor tendon on proximal interphalangeal joint}

At the site of proximal interphalangeal (PIP) joint, the enthesis of the central slip of the extensor tendon and the synovial fold and recess are intimately related. Central slip enthesitis (CSE), firstly described by Filippou et al, is defined as grey scale evidence of hypoechoic and thickened enthesis insertion, when compared to the proximal part of the tendon and to the controlateral joint [35]. The detection of CSE has been described to be usually in association with articular synovitis in PsA patients $[8,35]$, manifesting a typical example of hand's SEC inflammatory involvement (fig 7).

\section{Conclusions}

Integrating US with clinical evaluation to improve diagnostic accuracy can nowadays be considered an achievable target. In this perspective the identification of highly specific US elementary lesions (i.e. enthesitis) could be useful to assist the rheumatologist in the diagnosis and therapeutic strategies of arthritis.In the light of the crucial role of functional and classical entheses at hand level, and considering the hand involvement as one the most common presentation patterns of arthritis, the US assessment of those structures should become a routine standard in patients with arthritis and particularly in PsA.

\section{Conflict of interest: none}

\section{References}

1. Jacques P, Lambrecht S, Verheugen E, et al. Proof of concept: enthesitis and new bone formation in spondyloarthritis are driven by mechanical strain and stromal cells. Ann Rheum Dis 2014;73:437-445.

2. Lories RJ, Derese I, Luyten FP. Modulation of bone morphogenetic protein signaling inhibits the onset and progression of ankylosing enthesitis. J Clin Invest 2005;115:15711579 .

3. Ball J. Enthesopathy of rheumatoid and ankylosing spondylitis. Ann Rheum Dis 1971;30:2132-23.

4. McGonagle D, Lories RJ, Tan AL, Benjamin M. The concept of a "synovio-entheseal complex" and its implications for understanding joint inflammation and damage in psoriatic arthritis and beyond. Arthritis Rheum 2007;56:2482-291.

5. McGonagle D, Gibbon W, Emery P. Classification of inflammatory arthritis by enthesitis. Lancet 1998;352:11371140.

6. Mandl P, Navarro-Compan V, Terslev L, et al. EULAR recommendations for the use of imaging in the diagnosis and management of spondyloarthritis in clinical practice. Ann Rheum Dis 2015;74:1327-1339.

7. Zabotti A, Bandinelli F, Batticciotto A, et al. Musculoskeletal ultrasonography for psoriatic arthritis and psoriasis patients: a systematic literature review. Rheumatology (Oxford) 2017 May 18. doi:10.1093/rheumatology/kex179.

8. Zabotti A, Salvin S, Quartuccio L, De Vita S. Differentiation between early rheumatoid and early psoriatic arthritis by the ultrasonographic study of the synovio-entheseal complex of the small joints of the hands. Clin Exp Rheumatol 2016;34:459-465.

9. Gutierrez M, Filippucci E, Salaffi F, Di Geso L, Grassi W. Differential diagnosis between rheumatoid arthritis and psoriatic arthritis: The value of ultrasound findings at metacarpophalangeal joints level. Ann Rheum Dis 2011;70:1111-1114.

10. Fournié B, Margarit-Coll N, Champetier de Ribes TL, et al. Extrasynovial ultrasound abnormalities in the psoriatic finger. Prospective comparative power-doppler study versus rheumatoid arthritis. Joint Bone Spine 2006;73:527-531.

11. Benjamin M, McGonagle D. The anatomical basis for disease localisation in seronegative spondyloarthropathy at entheses and related sites. J Anat 2001;199:503-526.

12. McGonagle D, Marzo-Ortega H, Benjamin M, Emery P. Report on the Second international Enthesitis Workshop. Arthritis Rheum 2003;48:896-905.

13. Alcalde M, D’Agostino MA, Bruyn GA, et al. A systematic literature review of US definitions, scoring systems and validity according to the OMERACT filter for tendon lesion in RA and other inflammatory joint diseases. Rheumatology (Oxford) 2012;51:1246-1260.

14. Gutierrez M, Filippucci E, De Angelis R, Filosa G, Kane D, Grassi W. A sonographic spectrum of psoriatic arthritis: "the five targets." Clin Rheumatol 2010;29:133-42.

15. Macia C, Hammer HB, Falcao S, Medina J, Gutierrez M, De Miguel E. Reliability of sonographic peritendon extensor tendon inflammation pattern in psoriatic arthritis. Abstract EULAR 2017 AB1011. Ann Rheum Dis 2017;76 (Suppl 2).

16. Batticciotto A, Prato, Antivalle M. Potential role of metacarpophalangeal joints ultrasound in the differential diagnosis between early rheumatoid arthritis and early spondyloarthritis. Abstract EULAR 2017 SAT0620. Ann Rheum Dis 2017;76 (Suppl 2).

17. Guerini H, Pessis E, Theumann N, et al. Sonographic appearance of trigger fingers. J Ultrasound Med 2008;27:14071413.

18. Spirig A, Juon B, Banz Y, Rieben R, Vögelin E. Correlation Between Sonographic and In Vivo Measurement of A1 Pulleys in Trigger Fingers. Ultrasound Med Biol 2016;42:1482-1490.

19. Tan AL, Fukuba E, Halliday NA, Tanner SF, Emery P, McGonagle D. High-resolution MRI assessment of dactylitis in psoriatic arthritis shows flexor tendon pulley and sheathrelated enthesitis. Ann Rheum Dis 2015;74:185-189.

20. Tinazzi I, Marchetta A, Chessa D, McGonagle D, Macchioni P. Finger flexor tendon pulley complex involvement in PsA: 
an high resolution ultrasonographic study. Abstract EULAR 2017 SAT0454. Ann Rheum Dis 2017;76 (Suppl 2).

21. Geijer M, Lindqvist U, Husmark T, et al. The Swedish Early Psoriatic Arthritis Registry 5-year Followup: Substantial Radiographic Progression Mainly in Men with High Disease Activity and Development of Dactylitis. J Rheumatol 2015;42:2110-2117.

22. Bakewell CJ, Olivieri I, Aydin SZ, et al. Ultrasound and magnetic resonance imaging in the evaluation of psoriatic dactylitis: status and perspectives. J Rheumatol 2013;40:1951-1957.

23. Tuttle KS, Vargas SO, Callahan MJ, Bae DS, Nigrovic PA. Enthesitis as a component of dactylitis in psoriatic juvenile idiopathic arthritis: Histology of an established clinical entity. Pediatr Rheumatol Online J 2015;13:7.

24. Bandinelli F, Denaro V, Prignano F, Collaku L, Ciancio G, Matucci-Cerinic M. Ultrasonographic wrist and hand abnormalities in early psoriatic arthritis patients: Correlation with clinical, dermatological, serological and genetic indices. Clin Exp Rheumatol 2015;33:330-335.

25. Fournié B, Margarit-Coll N, Champetier de Ribes TL, et al. Extrasynovial ultrasound abnormalities in the psoriatic finger. Prospective comparative power-doppler study versus rheumatoid arthritis. Joint Bone Spine 2006;73:527-531.

26. Ash ZR, Tinazzi I, Gallego CC, et al. Psoriasis patients with nail disease have a greater magnitude of underlying systemic subclinical enthesopathy than those with normal nails. Ann Rheum Dis 2012;71:553-556.

27. Errichetti E, Zabotti A, Stinco G, et al. Dermoscopy of nail fold and elbow in the differential diagnosis of early psoriatic arthritis sine psoriasis and early rheumatoid arthritis. J Dermatol 2016;43:1217-1220.
28. Tan AL, Benjamin M, Toumi H, et al. The relationship between the extensor tendon enthesis and the nail in distal interphalangeal joint disease in psoriatic arthritis--a highresolution MRI and histological study. Rheumatology (Oxford) 2007;46:253-256.

29. Aydin SZ, Castillo-Gallego C, Ash ZR, et al. Ultrasonographic assessment of nail in psoriatic disease shows a link between onychopathy and distal interphalangeal joint extensor tendon enthesopathy. Dermatology 2012;225:231235 .

30. Terslev L, Naredo E, Iagnocco A, et al. Defining enthesitis in spondyloarthritis by ultrasound: results of a Delphi process and of a reliability reading exercise. Arthritis Care Res 2014;66:741-748.

31. Berritto D, Iacobellis F, Rossi C, Reginelli A, Cappabianca S, Grassi R. Ultra high-frequency ultrasound: New capabilities for nail anatomy exploration. J Dermatol 2017;44:4346.

32. Sandobal C, Carbó E, Iribas J, Roverano S, Paira S. Ultrasound nail imaging on patients with psoriasis and psoriatic arthritis compared with rheumatoid arthritis and control subjects. J Clin Rheumatol 2014;20:21-24.

33. McGonagle D. Enthesitis: an autoinflammatory lesion linking nail and joint involvement in psoriatic disease. J Eur Acad Dermatol Venereol 2009;23 Suppl 1:9-13.

34. Gisondi P, Idolazzi L, Girolomoni G. Ultrasonography reveals nail thickening in patients with chronic plaque psoriasis. Arch Dermatol Res 2012;304:727-732.

35. Filippou G, Di Sabatino V, Adinolfi A, et al. No enthesis should be overlooked when psoriatic arthritis is suspected: Enthesitis of the extensor digitorum tendons. J Rheumatol 2013;40:335. 